\title{
Stability Analysis for Uncertain Delayed T-S Fuzzy Systems
}

\author{
Jiali Yu \\ School of Mathematical Sciences \\ University of Electronic Science and Technology of China \\ Chengdu, P. R. China. \\ e-mail: yujiali@uestc.edu.cn
}

\author{
Yong Liao \\ School of Computer Science and Engineering \\ University of Electronic Science and Technology of China \\ Chengdu, P. R. China. \\ e-mail: Liaoyong@uestc.edu.cn
}

\begin{abstract}
This paper studies the global exponential stability for a Takagi-Sugeno (T-S) fuzzy system with bounded uncertain delays. Most existing T-S method represent global nonlinear systems by connecting local linear systems with linguistic description. However, many complex systems cannot be represented by linear systems. In this paper, a class of local nonlinear systems having nice dynamic properties is employed to represent some global complex systems. Moreover, the delays are any uncertain bounded continuous functions. Sufficient conditions for global exponential stability of these delayed global complex systems are derived. Criteria for design of nonlinear fuzzy controllers to feedback control the stability of global nonlinear fuzzy systems are given.

Keywords-Fuzzy systems; Global exponential stability; Delays
\end{abstract}

\section{INTRODUCTION}

Since Tanaka and Sugeno [1] proposed Takagi-Sugeno (TS) fuzzy model in 1985, a great number of results have been reported for T-S systems [2], [3], [4], [5]. The T-S model gives an effective method to combine some simple local systems with their linguistic description to represent complex nonlinear dynamic systems. Stability of T-S model fuzzy systems is quite important for practical applications. It has been widely studied by many authors, see, for examples, [6], [7], [8].

Time delays in dynamic systems has been studied for many years. T-S model of fuzzy systems with delays was first introduced in [9]. It is well known that delays can affect dynamics of some nonlinear systems, a stable system may become unstable by introducing some delays [10]. In recent years, some authors have paid their attention to control of nonlinear systems with delays by using T-S fuzzy models. There exist two kinds of delays, one is continuous, see, for examples [11], [12], [13], [14]. The other is discrete, see, for examples, [15], [16]. In control engineering, delays are difficult to be known exactly, so stability for systems with uncertain delays are quite interesting [17]. In this paper, the delays are assumed to be any uncertain bounded continuous functions. Stability conditions will be derived, some of them will be represented by simple algebraic inequalities and are easy to check.

As we know, in most reported stability results of T-S model, simple linear systems are used to form global nonlinear fuzzy systems [18], [19], [20]. However, there are many complex nonlinear fuzzy systems cannot be connected by using local linear systems. In this paper, unlike using local linear systems in previous study, a class of nonlinear systems with delays having nice dynamical properties [21] will be used as local systems to form some global complex nonlinear fuzzy systems by T-S method. Since global exponential stability (GES) is more interesting than global asymptotic stability, Our stability conditions will guarantee the global exponential stability of the global complex nonlinear fuzzy delayed systems.

This paper is organized as follows. In Section II, some preliminaries for delayed fuzzy control systems will be given. In Section III, conditions for global exponential stability of fuzzy systems with delays will be proposed and proved. In Section IV, simulations will be given. This paper will be concluded in Section V.

\section{PRELIMINARIES}

Consider a T-S fuzzy time-delay model which is composed of $r$ plant rules. For each $s=1, \cdots, r$, the $s$ th plant rule can be represented as follows: THEN

Plant Rule $s$ : IF $\alpha_{1}(t)$ is $M_{1 s}$ AND $\cdots$ AND $\alpha_{p}(t)$ is $M_{p s}$

$$
\dot{x}(t)=-x(t)+W_{s} g(x(t))+J_{s} g\left(x\left(t-\tau_{s}(t)\right)\right)
$$

for $t \geq 0$, where $x(t)=\left(x_{1}(t), \cdots, x_{n}(t)\right)^{T}$ is the state vector, $r$ is the number of IF-THEN rules, $\alpha_{1}(t), \cdots, \alpha_{p}(t)$ are the premise variables and each $M_{i s}(i=1, \cdots, p)$ is the fuzzy set corresponding to $\alpha_{i}(t)$ and plant rule $s . u(t)$ is the control input vector, $\tau_{s}(t)$ is the time delay which satisfies $0 \leq \tau_{s}(t) \leq$ $\tau$.

For any $x \in R^{n}, g(x)=\left(g\left(x_{1}\right), \cdots, g\left(x_{n}\right)\right)^{T}$, and the function $g$ is defined as follows:

$$
g(s)=\frac{|s+1|-|s-1|}{2}, \quad s \in R .
$$

The function $g$ is continuous but non-differentiable. So the local system is nonlinear which is the main feature of this paper different from others.

The system of (1) can be described as follows:

$$
\begin{aligned}
\dot{x}_{i}(t)= & -x_{i}(t) \\
& +\sum_{j=1}^{n}\left[W_{i j}^{s} g\left(x_{j}(t)\right)+J_{i j}^{s} g\left(x_{j}\left(t-\tau_{s}(t)\right)\right)\right]
\end{aligned}
$$


for $t \geq 0$ and $i=1, \cdots, n$. Where $W_{s}=\left(W_{i j}^{s}\right)_{n \times n}, J_{s}=$ $\left(J_{i j}^{s}\right)_{n \times n}$ is the constant matrix.

Let $M_{i s}\left(\alpha_{i}(t)\right)$ be the membership function of the fuzzy set $M_{i s}$ at the position $\alpha_{i}(t)$ and denote

$$
\begin{gathered}
w_{s}(\alpha(t))=\prod_{i=1}^{p} M_{i s}\left(\alpha_{i}(t)\right), \\
h_{s}(\alpha(t))=\frac{w_{s}(\alpha(t))}{\sum_{i=1}^{r} w_{i}(\alpha(t))} \geq 0 \\
\sum_{s=1}^{r} h_{s}(\alpha(t))=1 .
\end{gathered}
$$

Then the overall delayed fuzzy control system is inferred as

$$
\begin{aligned}
\dot{x}(t)= & \sum_{s=1}^{r} h_{s}(\alpha(t))\left[-x(t)+W_{s} g(x(t))\right. \\
& \left.+J_{s} g\left(x\left(t-\tau_{s}(t)\right)\right)\right]
\end{aligned}
$$

for $t \geq 0$.

For each solution, the initial value is assumed to be

$$
x(t)=\phi(t), \quad t \in[-\tau, 0]
$$

where $\phi(t)=\left(\phi_{1}(t), \cdots, \phi_{n}(t)\right)^{T}$ is a vector continuous function. We define

$$
\|\phi\|=\sup _{-\tau \leq \theta \leq 0} \sqrt{\phi_{1}^{2}(\theta)+\cdots+\phi_{n}^{2}(\theta)} .
$$

In this paper, for a matrix $S$, we will use $S>0$ and $S<0$ to denote that $S$ is a symmetric positive matrix or a symmetric negative matrix, respcetively.

$D^{+}$is used to denote the upper righthand Dini derivative in this paper. For any continuous function $g: R \rightarrow R$, the upper righthand Dini derivative of $g(t)$ is defined as

$$
D^{+} g(t)=\lim _{\theta \rightarrow 0^{+}} \sup \frac{g(t+\theta)-g(t)}{\theta} .
$$

It is easy to see that if $g(t)$ is locally Lipschitz then $\left|D^{+} g(t)\right|<$ $+\infty$.

\section{Stability ANALysis OF FUZZy Delayed Systems}

Consider the fuzzy system (3), we can see that it is a global nonlinear fuzzy system and its nonlinear local delayed systems are represented as follows

$$
\dot{x}(t)=-x(t)+W_{s} g(x(t))+J_{s} g\left(x\left(t-\tau_{s}(t)\right)\right)
$$

for $t \geq 0$.

In [17], we can see that the fuzzy system (3) is globally exponentially stable, if there exist constants $\epsilon>0$ and $\Pi \geq 1$ such that

$$
\|x(t)\| \leq \Pi\|\phi\| e^{-\epsilon t}
$$

for all $t \geq 0$.

In the following Theorem, we will derive some global exponential stability conditions which will be presented in some simple algebraic inequalities.
Theorem 1: If

$$
-1+W_{i i}^{s}+\sum_{j=1}^{n}\left[\left|W_{i j}^{s}\right|\left(1-\delta_{i j}\right)+\left|J_{i j}^{s}\right|\right]<0
$$

for all $i=1, \cdots, n$ and $s=1, \cdots, r$, where

$$
\delta_{i j}= \begin{cases}1, & i=j \\ 0, & i \neq j\end{cases}
$$

then, the free fuzzy system (3) is globally exponentially stable. proof For any delays $\tau_{s}(t)(s=1, \cdots, r)$, since $0 \leq \tau_{s}(t) \leq$ $\tau$, the free fuzzy system of (3) can be rewritten as

$$
\begin{aligned}
\dot{x}_{i}(t)= & -x_{i}(t)+\sum_{s=1}^{r} h_{s}(\alpha(t))\left[\sum _ { j = 1 } ^ { n } \left(W_{i j}^{s} g\left(x_{j}(t)\right)\right.\right. \\
& \left.\left.+J_{i j}^{s} g\left(x_{j}\left(t-\tau_{s}(t)\right)\right)\right)\right] .
\end{aligned}
$$

Then, it follows that

$$
\begin{aligned}
D^{+}\left|x_{i}(t)\right| \leq & -\left|x_{i}(t)\right|+\sum_{s=1}^{r} h_{s}(\alpha(t))\left[W_{i i}^{s}\left|g\left(x_{i}(t)\right)\right|\right. \\
& +\sum_{j=1}^{n}\left(\left|W_{i j}^{s}\right|\left(1-\delta_{i j}\right)\left|g\left(x_{j}(t)\right)\right|\right. \\
& \left.\left.+\left|J_{i j}^{s}\right|\left|g\left(x_{j}\left(t-\tau_{s}(t)\right)\right)\right|\right)\right]
\end{aligned}
$$

for all $t \geq 0$.

Since

$$
-1+W_{i i}^{s}+\sum_{j=1}^{n}\left[\left|W_{i j}^{s}\right|\left(1-\delta_{i j}\right)+\left|J_{i j}^{s}\right|\right]<0,
$$

then there must exist a $\epsilon>0$ such that

$$
\epsilon-1+W_{i i}^{s}+\sum_{j=1}^{n}\left[\left|W_{i j}^{s}\right|\left(1-\delta_{i j}\right)+e^{\epsilon \tau}\left|J_{i j}^{s}\right|\right]<0 .
$$

Denote

$$
\eta_{i s}=-\left[\epsilon-1+W_{i i}^{s}+\sum_{j=1}^{n}\left[\left|W_{i j}^{s}\right|\left(1-\delta_{i j}\right)+e^{\epsilon \tau}\left|J_{i j}^{s}\right|\right]\right]
$$

and let

$$
\sigma=\min _{1 \leq i \leq n, 1 \leq s \leq r}\left(\eta_{i s}\right)
$$

Obviously, $\sigma>0$. Define $z_{i}(t)=\left|x_{i}(t)\right| e^{\epsilon t},(i=1, \cdots, n)$ for all $t \geq-\tau$. Then, it follows from (6) that

$$
\begin{aligned}
D^{+} z_{i}(t) \leq & (\epsilon-1)\left|x_{i}(t)\right| e^{\epsilon t}+\sum_{s=1}^{r} h_{s}(\alpha(t))\left[W_{i i}^{s}\left|g\left(x_{i}(t)\right)\right|\right. \\
& +\sum_{j=1}^{n}\left(\left|W_{i j}^{s}\right|\left(1-\delta_{i j}\right)\left|g\left(x_{j}(t)\right)\right|\right. \\
& \left.\left.+\left|J_{i j}^{s}\right|\left|g\left(x_{j}\left(t-\tau_{s}(t)\right)\right)\right|\right)\right] e^{\epsilon t}
\end{aligned}
$$

for all $t \geq 0$.

From the definition of function $g$, we can see that $\left|g\left(x_{i}(t)\right)\right| \leq\left|x_{i}(t)\right|,(i=1, \cdots, n)$. 
So,

$$
\begin{aligned}
D^{+} z_{i}(t) \leq & (\epsilon-1)\left|x_{i}(t)\right| e^{\epsilon t}+\sum_{s=1}^{r} h_{s}(\alpha(t))\left[W_{i i}^{s}\left|x_{i}(t)\right|\right. \\
& +\sum_{j=1}^{n}\left(\left|W_{i j}^{s}\right|\left(1-\delta_{i j}\right)\left|x_{j}(t)\right|\right. \\
& \left.\left.+\left|J_{i j}^{s}\right|\left|x_{j}\left(t-\tau_{s}(t)\right)\right|\right)\right] e^{\epsilon t} \\
\leq & (\epsilon-1) z_{i}(t)+\sum_{s=1}^{r} h_{s}(\alpha(t))\left[W_{i i}^{s} z_{i}(t)\right. \\
& +\sum_{j=1}^{n}\left(\left|W_{i j}^{s}\right|\left(1-\delta_{i j}\right) z_{j}(t)\right. \\
& \left.\left.+e^{\epsilon \tau}\left|J_{i j}^{s}\right| z_{j}\left(t-\tau_{s}(t)\right)\right)\right] \\
& \leq \sum_{s=1}^{r} h_{s}(\alpha(t))\left[\left(-1+W_{i i}^{s}+\epsilon\right) z_{i}(t)\right. \\
& +\sum_{j=1}^{n}\left(\left|W_{i j}^{s}\right|\left(1-\delta_{i j}\right) z_{j}(t)\right. \\
& \left.\left.+e^{\epsilon \tau}\left|J_{i j}^{s}\right| z_{j}\left(t-\tau_{s}(t)\right)\right)\right]
\end{aligned}
$$

for all $t \geq 0$.

For any constant $a>1$, it is easy to see that

$$
z_{i}(t)=\left|\phi_{i}(t)\right| e^{\epsilon t} \leq\|\phi\|<a\|\phi\|
$$

for all $t \in[-\tau, 0]$. We will prove that $z_{i}(t)<a\|\phi\|(i=$ $1, \cdots, n)$ for all $t \geq 0$. Otherwise, then there must exist some $i$ and a time $t_{1}>0$ such that

$$
z_{i}\left(t_{1}\right)=a\|\phi\|
$$

and

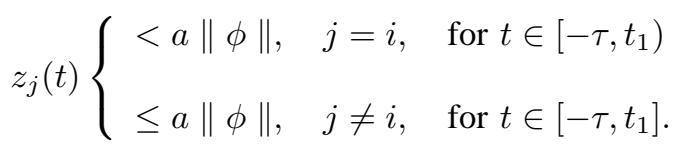

Then, we have $D^{+} z_{i}\left(t_{1}\right) \geq 0$. But on the other hand, it follows from (7) that

$$
\begin{aligned}
D^{+} z_{i}\left(t_{1}\right) \leq & \sum_{s=1}^{r} h_{s}\left(\alpha\left(t_{1}\right)\right)\left[\left(-1+W_{i i}^{s}+\epsilon\right) a\|\phi\|\right. \\
& \left.+a\|\phi\| \sum_{j=1}^{n}\left(\left|W_{i j}^{s}\right|\left(1-\delta_{i j}\right)+e^{\epsilon \tau}\left|J_{i j}^{s}\right|\right)\right] \\
= & -a\|\phi\| \sum_{s=1}^{r} h_{s}\left(\alpha\left(t_{1}\right)\right) \cdot \eta_{i s} \\
\leq & -\sigma a\|\phi\| \\
< & 0 .
\end{aligned}
$$

This is a contradiction and it proves that $z_{i}(t)<a\|\phi\|(i=$ $1, \cdots, n)$ for all $t \geq 0$.

Letting $a \rightarrow 1$, we have $z_{i}(t) \leq\|\phi\|$ for all $t \geq 0$. Then, it follows that

$$
\left|x_{i}(t)\right| \leq\|\phi\| e^{-\epsilon t}
$$

for all $t \geq 0$.

The proof is complete.

The above theorem provide some conditions to guarantee the exponential stability of the free fuzzy systems of (3) subject to any uncertain continuous bounded delays.

\section{Simulations}

In this section, we will give an example to illustrate the above theory.

Consider the following nonlinear system with delay

$$
\left\{\begin{aligned}
\dot{x}_{1}(t)= & -x_{1}(t)-g\left(x_{1}(t)\right) \cdot\left(1+\sin ^{2} x_{2}(t)\right) \\
& +g\left(x_{1}(t-\tau(t))\right)+g\left(x_{2}(t-\tau(t))\right) \cdot \sin ^{2} x_{2}(t) \\
\dot{x}_{2}(t)= & -x_{2}(t)-g\left(x_{2}(t)\right) \\
& +\left(g\left(x_{1}(t)\right)-g\left(x_{2}(t)\right)\right) \cdot \cos ^{2} x_{2}(t) \\
& +g\left(x_{1}(t-\tau(t))\right) \cdot \cos ^{2} x_{2}(t) \\
& +g\left(x_{2}(t-\tau(t))\right) \cdot \sin ^{2} x_{2}(t)
\end{aligned}\right.
$$

for all $t \geq 0$.

Define some matrices

$$
\begin{array}{ll}
W_{1}=\left(\begin{array}{cc}
-2 & 0 \\
0 & -1
\end{array}\right), & J_{1}=\left(\begin{array}{ll}
1 & 1 \\
0 & 1
\end{array}\right) \\
W_{2}=\left(\begin{array}{cc}
-1 & 0 \\
1 & -2
\end{array}\right), & J_{2}=\left(\begin{array}{ll}
1 & 0 \\
1 & 0
\end{array}\right)
\end{array}
$$

and some functions

$$
M_{11}\left(x_{2}(t)\right)=\sin ^{2} x_{2}(t), \quad M_{22}\left(x_{2}(t)\right)=\cos ^{2} x_{2}(t) .
$$

We can interpret $M_{11}\left(x_{2}(t)\right)$ and $M_{22}\left(x_{2}(t)\right)$ as membership functions of some fuzzy sets $M_{11}$ and $M_{22}$, respectively. Using these fuzzy sets, the above nonlinear system (8) can be presented by the following TS fuzzy model

Plant Rule 1: IF $x_{2}(t)$ is $M_{11}$ THEN

$$
\dot{x}(t)=-x(t)+W_{1} g(x(t))+J_{1} g(x(t-\tau(t))) .
$$

Plant Rule 2: IF $x_{2}(t)$ is $M_{22}$ THEN

$$
\dot{x}(t)=-x(t)+W_{2} g(x(t))+J_{2} g(x(t-\tau(t))) .
$$

Using the Theorem 1, it is easy to check that the nonlinear system (8) is globally exponentially stable. The delay $\tau(t)$ could be any bounded continuous function, say, $\sin ^{2}(t)$, $\cos ^{2}(t), 1 /(1+|t|)$ (not differentiable), etc.

Fig. 1 shows the global exponential stability of the nonlinear system (8) with $\tau(t)=1$.

Fig. 2 and Fig. 3 show the global exponential stability of the local systems (9) and (10) with $\tau(t)=1$.

\section{Conclusions}

In this paper, the global exponential stability analysis for a class of fuzzy systems with uncertain time delays has been studied. Some global exponential stability conditions for free delayed fuzzy systems have been proposed. Then an example has been used to illustrate the results. We believe that all of the results obtained in this paper can be extended to the fuzzy systems with multiple time delays or with time-varying delay. 


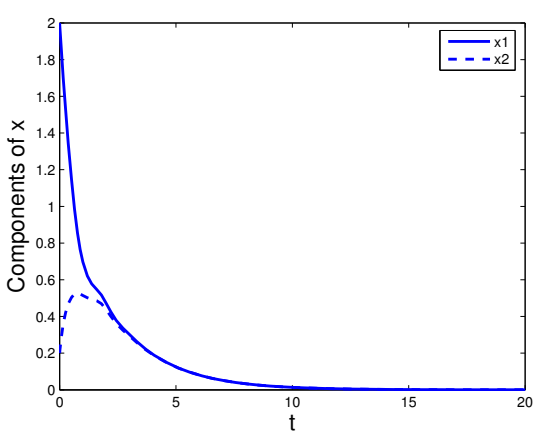

Fig. 1. Global exponential stability of (8) with $\tau(t)=1$.

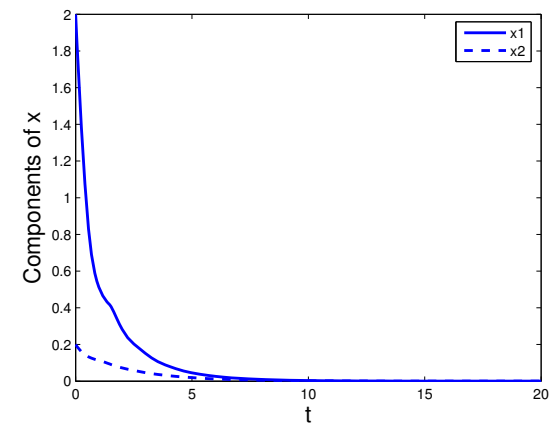

Fig. 2. Global exponential stability of (9) with $\tau(t)=1$.

\section{ACKNOWLEDGMENT}

This work is supported in part by the National Natural Science Foundation of China under Grant 61103041, 11071178, the Fundamental Research Funds for the Central Universities, under Grant ZYGX2013Z005, ZYGX2012J070, the National High-tech R\&D Program under Grant SQ2011GX02D03708.

\section{REFERENCES}

[1] T. Takagi and M. Sugeno, Fuzzy identification of systems and its applications to modeling and control, IEEE Trans. Syst., Man, Cybern., vol. 15 , no. 1 , pp. $116-132,1985$.

[2] F. H. Hsiao, C. W. Chen, Y. W. Liang, S. D. Xu and W. L. Chiang, T-S fuzzy controllers for nonlinear Interconnected systems with multiple time delays, IEEE Trans. Circuits And Systems-I, vol. 52, no. 9, pp. 1883-1893, 2005.

[3] F. Liu, M. Wu, Y. He and R. Yokoyama, New delay-dependent stability criteria for TCS fuzzy systems with time-varying delay, Fuzzy Sets and Systems, vol. 161, pp. 2033-2042, 2010.

[4] X. Su, P. Shi, L. Wu and Y. Song, A Novel Approach to Filter Design for TCS Fuzzy Discrete-Time Systems With Time-Varying Delay, IEEE Trans. Fuzzy Systems, vol. 20, no. 6, pp. 1114-1129, 2012.

[5] J. Yu, Z. Yi, and L. Zhang, Periodicity of a class of nonlinear fuzzy systems with delays, Chaos, Solitons and Fractals, vol. 40, pp. $1343-$ 1351, 2009.

[6] K. Tanaka, Stability and stabilizability of fuzzy neural linear control systems, IEEE Trans. Fuzzy Systems, vol. 3, pp. 438-447, 1995.

[7] M. A. L. Thathachar and P. Viswanath, On the stability of fuzzy systems, IEEE Trans. Fuzzy Systems, vol. 5, pp. 145-151, 1997.

[8] F. Cuesta, F. Gordillo, J. Aracil and A. Ollero, Stability analysis of nonlinear multivariable Takagi-Sugeno fuzzy control systems,IEEE Trans. Fuzzy Systems, vol. 7, pp. 508-520, 1999.

[9] Y. Y. Cao and P. M. Frank, Analysis and synthesis of nonlinear time-delay systems via fuzzy control approach, IEEE Trans. Fuzzy Systems, vol. 8 , no. 2, pp. 200-211, 2000.

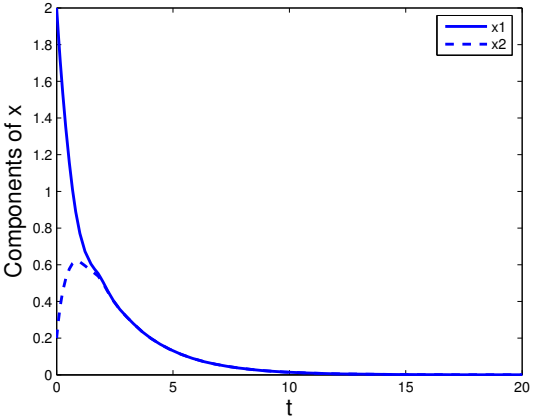

Fig. 3. Global exponential stability of (10) with $\tau(t)=1$.

[10] P. Baldi, A. F. Atiya, How delays affect neural dynamics and learning, IEEE Trans. Neural Networks, vol. 5, no. 4, pp. 612-621, 1994.

[11] F. H. Hsiao, W. L. Chiang and C. W. Chen, Application of fuzzy $H_{\infty}$ control via T-S fuzzy models for nonlinear time-delay systems, Int. J. Art. Intell. Tools, vol. 12, no. 2, pp. 117-137, 2003.

[12] E. Fridman and U. Shaked, Parameter dependent stability and stability and stabilization of uncertain time-delay systems, IEEE Trans. Autom Control, vol. 48, no. 5, pp. 861-866, May 2003.

[13] X. P. Guan and C. L. Chen, Delay-dependent guaranteed cost control for T-S fuzzy systems with time delays, IEEE Trans. Fuzzy Systems, vol. 12, no. 2, pp. 236-249, 2004.

[14] I. Said, E. H. Tissir, Delay Dependent Robust Stability of T-S Fuzzy Systems with Additive Time Varying Delays, Applied Mathematical Sciences, Vol. 6, no. 1, pp. 1-12, 2012.

[15] S. S. Chen ,Y. C. Chang, S. F. Su, S. L. Chung and T. T. Lee, Robust static output-feedback stabilization for nonlinear discrete-time systems with time delay via fuzzy control approach, IEEE Trans. Fuzzy Systems, vol. 13 , no. 2, pp. 263-272, 2005

[16] S. Y. Xu and J. Lam, Robust $H^{\infty}$ control for uncertain discrete-timedelay fuzzy systems via output feedback controllers, IEEE Trans. Fuzzy Systems, vol. 13, no. 1, pp. 82-93, 2005

[17] Z. Yi and P. A. Heng, Stability of fuzzy control systems with bounded uncertain delays, IEEE Trans. Fuzzy Systems, vol. 10, no. 1, pp. 92-97, 2002.

[18] C. L. Chen, G. Feng and X. P. Guan, Delay-dependent stability analysis and controller synthesis for discrete-time T-S fuzzy systems with time delays, IEEE Trans. Fuzzy Systems, vol. 13, no. 5, pp. 630-643, 2005.

[19] Y. Zhao, H. Gao, J. Lam and B. Du, Stability and Stabilization of Delayed TCS Fuzzy Systems: A Delay Partitioning Approach, IEEE Trans. Fuzzy Systems, vol. 17, no. 4, pp. 750-762, 2009.

[20] X. P. Guan and C. L. Chen, Delay-dependent guaranteed cost control for T-S fuzzy systems with time delays, IEEE Trans. Fuzzy Systems, vol. 12, no. 2, pp. 236-249, 2004.

[21] Z. Yi and K. K. Tan, Convergence analysis of recurrent neural networks, Kluwer Academic Publishers, Boston, 2004. 